\title{
Recent results from NA61/SHINE
}

\author{
Seweryn Kowalski* \\ for the NA61/SHINE collaboration \\ Institute of Physics, University of Silesia, Katowice, Poland \\ E-mail: seweryn.kowalski@us.edu.pl
}

\begin{abstract}
The aim of the NA61/SHINE ion programme is to explore the QCD phase diagram within the range of thermodynamical variables accessible by the SPS. Moreover the experiment provides precision hadron production measurements to improve computation of neutrino fluxes in neutrino oscillation experiments and for improving air shower simulations of cosmic-ray experiments.

The main physics goals of the NA61/SHINE ion programme are the study of the properties of the onset of deconfinement and the search for signatures of the critical point of strongly interacting matter. These goals are pursued by performing an energy (beam momentum $13 \mathrm{~A}-158 \mathrm{~A} \mathrm{GeV/c}$ ) and system size (p+p, p+Pb, Be+Be, $\mathrm{Ar}+\mathrm{Sc}, \mathrm{Xe}+\mathrm{La})$ scan.
\end{abstract}

This paper reviews the status and results of the NA61/SHINE experiment.

9th International Workshop on Critical Point and Onset of Deconfinement - CPOD2014,

17-21 November 2014

ZiF (Center of Interdisciplinary Research), University of Bielefeld, Germany

\footnotetext{
*Speaker.
} 


\section{NA61/SHINE facility at the CERN SPS}

\subsection{The NA61/SHINE detector}

The layout of the NA61/SHINE detector [1] is sketched in Figure 1. It consists of a large acceptance hadron spectrometer with excellent capabilities in charged particle momentum measurements and identification by a set of six Time Projection Chambers as well as Time-of-Flight detectors. The high resolution forward calorimeter, the Projectile Spectator Detector, measures energy flow around the beam direction, which in nucleus-nucleus reactions is primarily a measure of the number of spectator (non-interacted) nucleons and thus related to the centrality of the collision. For hadron-nucleus interactions, the collision centrality is determined by counting low momentum particles emitted from the nuclear target with the LMPD detector (a small TPC) surrounding the target. An array of beam detectors identifies beam particles, secondary hadrons and ions as well as primary ions, and measures precisely their trajectories.

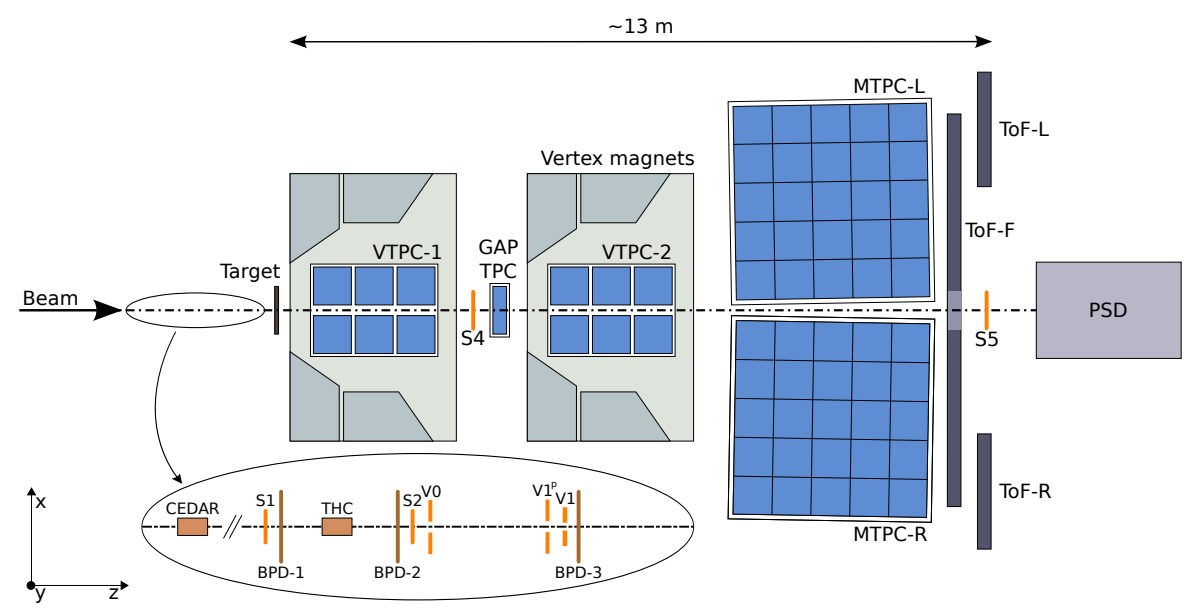

Figure 1: Schematic layout of the NA61/SHINE experiment at the CERN SPS (horizontal cut in the beam plane, not to scale).

\subsection{Beams for NA61/SHINE experiment}

The CERN accelerator chain is shown in Figure 2, higlighting the components relevant for NA61/SHINE. From the source, the beams of ions and protons pass through a series of accelerators, before they reach the SPS for final acceleration and subsequent extraction to the North Area and the NA61/SHINE experiment. The protons and ions follow a different path in the pre-injector chain to the PS, required to match the beam parameters for their acceleration. The proton beam is generated from hydrogen gas by a duo-plasmatron ion source and a focused and bunched beam is injected into LINAC2. The $50 \mathrm{MeV}$ proton beam from LINAC2 is then distributed in the four rings of the PS booster (PSB). After acceleration to $1.4 \mathrm{GeV}$ the beam is sent to the Proton Synchrotron (PS). The PS accelerates the beams to $14 \mathrm{GeV} / \mathrm{c}$ for injection into the Super Proton Synchrotron (SPS). In the SPS, the $14 \mathrm{GeV} / \mathrm{c}$ beam is accelerated to $400 \mathrm{GeV} / \mathrm{c}$.

The ions are generated in the ECR source. The ions which were ionized to a particular charge state enter the LINAC3 accelerator. From LINAC3 the beam is injected into the Low Energy Ion 


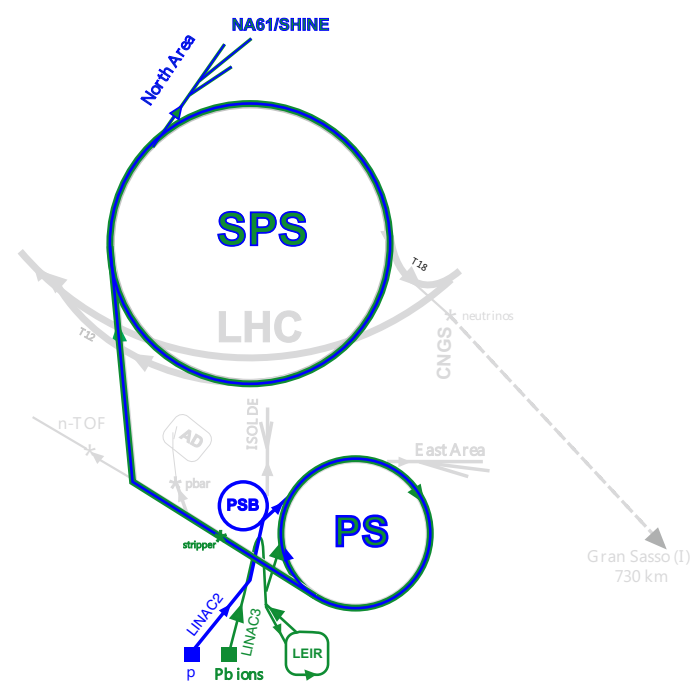

Figure 2: Schematic layout of the CERN accelerator complex relevant for the NA61/SHINE ion and proton beam operation (top view, not to scale).

Ring (LEIR) and accelerated to $72 \mathrm{MeV} / \mathrm{u}$. From LEIR the beam is injected into the PS machine. The extracted beam from the PS is accelerted by the SPS and is then slowly extracted to the North Area. The acceleration range in the SPS varies between $13 \mathrm{GeV} / \mathrm{u}$, which is the lowest possible operational range due to stability reasons, and $160 \mathrm{GeV} / \mathrm{u}$ due to limits in the power supplies and energy dissipation in the magnets.

The extracted beam from the SPS is transported over about $1 \mathrm{~km}$ by bending and focusing magnets and then split into three parts each one directed towards a primary target where secondary particles are created.

The NA61/SHINE experiment up to now used secondary hadron beams in the momentum range from $13 \mathrm{GeV} / \mathrm{c}$ to $350 \mathrm{GeV} / \mathrm{c}$, as well as attenuated primary Pb82+ ion beams in the energy range from $13 \mathrm{~A} \mathrm{GeV}$ to $158 \mathrm{~A} \mathrm{GeV}$. For the start of the ion physics program secondary ${ }^{7} \mathrm{Be}$ ions were produced via fragmentation of the $\mathrm{Pb} 82+$ ions as primary ions other than $\mathrm{Pb} 82+$ were not available before 2015 . The secondary ${ }^{7} \mathrm{Be}$ ions were produced via fragmentation of the $\mathrm{Pb} 82+$ ions. Starting in 2015 primary ${ }^{40} \mathrm{Ar}$ and ${ }^{131} \mathrm{Xe}$ ion beams in the same momentum range will be delivered to NA61/SHINE. In Figure 3 a charge spectrum of the Pb82+ fragmentation beam is presented showing secondary ${ }^{7} \mathrm{Be}$ ions together with other fragments which accompanied the "wanted ${ }^{7} \mathrm{Be}$ beam".

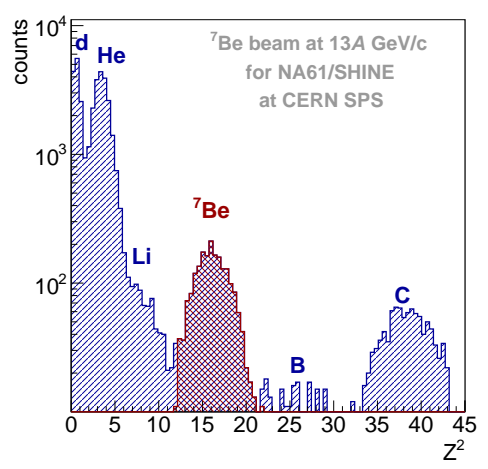

Figure 3: Charge spectrum measured with the Quartz Z-detector for $13 A \mathrm{GeV} / \mathrm{c} \mathrm{Pb} 82+$ fragmentation beam. 


\section{Particle identification methods}

Four different particle identification methods are used:

- for $\pi^{-}$spectra the $h^{-}$method [2], which is based on fact that the majority of negatively charged particles are $\pi^{-}$mesons. The contribution of other particles is subtracted using simulations based on models.

- for $\pi^{+}, \pi^{-}, K^{+}, K^{-}$and $p, \bar{p}$ the specific energy loss (the $d E / d x$ method), which uses the energy loss in the TPC gas to identify particles. In each bin of $p, p_{T}$ and charge a sum of Gauss functions is fitted to the $d E / d x$ spectrum. For each track its Likelihood value for being a given hadron is calculated based on the fitted $d E / d x$ distribution.

- in addition to the $d E / d x$ information time of flight measurements are used in the tof $-d E / d x$ method. The time-of-flight measurement together with the particle path length and momentum measured by the TPCs allow to calculate its mass $m$. The tof information allows the extension of identification into phase space regions near mid-rapidity where $d E / d x$ identification becomes ambiguous.

- the $\Lambda$ hyperon analysis can be considered as an example of the $V^{0}$ particles identification method. The analysis was performed using the main decay channel $\Lambda \rightarrow p+\pi^{-}+X$, showing up as a characteristic $V^{0}$ pattern (instant appearance of a positive and a negative track) being searched for by a dedicated pattern reconstruction algorithm. The raw number of $\Lambda$ hyperons is extracted by fitting invariant mass distributions by a sum of Lorentzian (signal) and a polynomial of $3^{\text {rd }}$ order (background).

The results of each of these method are corrected for particles originating from weak decays and secondary interactions (feed-down) and detector effects (acceptance, efficiency, ...) using particle production models and a simulation of the detector.

\section{Results from $\mathrm{p}+\mathrm{p}$ and ${ }^{7} \mathrm{Be}+{ }^{9} \mathrm{Be}$ collisions}

\subsection{Inelastic ${ }^{7} \mathrm{Be}+{ }^{9} \mathrm{Be}$ cross section}

In the data taking on ${ }^{7} \mathrm{Be}+{ }^{9} \mathrm{Be}$ collisions in 2012/2013 a $2 \mathrm{~cm}$ diameter interaction trigger counter S4 was placed on the beam-line between VTPC-1 and VTPC -2 . The primary purpose was to reduce the background of downstream interactions by requiring that the incoming ${ }^{7} \mathrm{Be}$ nucleus had interacted in the ${ }^{9} \mathrm{Be}$ target. This arrangement also offered the possibility to measure the total inelastic ${ }^{7} \mathrm{Be}+{ }^{9} \mathrm{Be}$ cross section $\sigma_{\text {inel }}$ [3]. The values measured by NA61/SHINE at $13 \mathrm{~A}, 20 \mathrm{~A}$ and $30 A \mathrm{GeV} / \mathrm{c}$ are plotted in Figure 4. The NA61/SHINE results are in good agreement with an earlier measurement at lower beam momentum [4] and a calculation using the Glissando model [5].

\subsection{Pion spectra}

Preliminary double differential spectra (in cms rapidity y and transverse momentum $p_{T}$ ) of negatively charged pions produced in Be+Be collisions at $40 \mathrm{~A}, 75 \mathrm{~A}$ and $150 \mathrm{~A} \mathrm{GeV/c}$ were obtained using the $h^{-}$method. The spectra were obtained in four centrality classes: $0-5 \%, 5-10 \%$, 


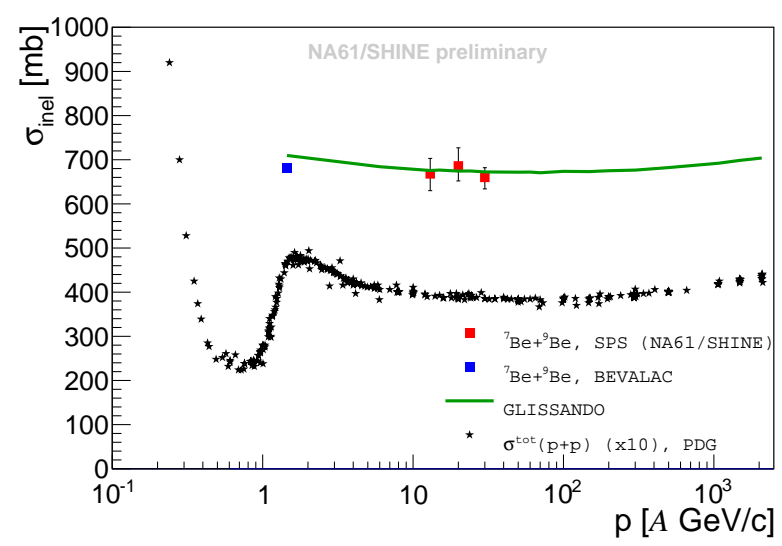

Figure 4: Total inelastic cross section $\sigma_{\text {inel }}$ versus beam momentum for ${ }^{7} \mathrm{Be}+{ }^{9} \mathrm{Be}$ interactions measured by NA61/SHINE (red squares) and LBNL (blue square) [4]. Calculations using the Glissando model [5] are shown by the green curve for comparison.

$10-15 \%$, and $15-20 \%$. The centrality is determined as a fraction of total inelastic cross-section obtained from the measured PSD energy spectrum. Example results for the first centrality class $(0-5 \%)$ are presented in Figure 5. For comparison the double differential inclusive spectra of $\pi^{-}$ mesons in rapidity and transverse momentum produced in inelastic $p+p$ interactions at 20, 31, 40, 80 and $158 \mathrm{GeV} / \mathrm{c}$ are shown in Figure 6.
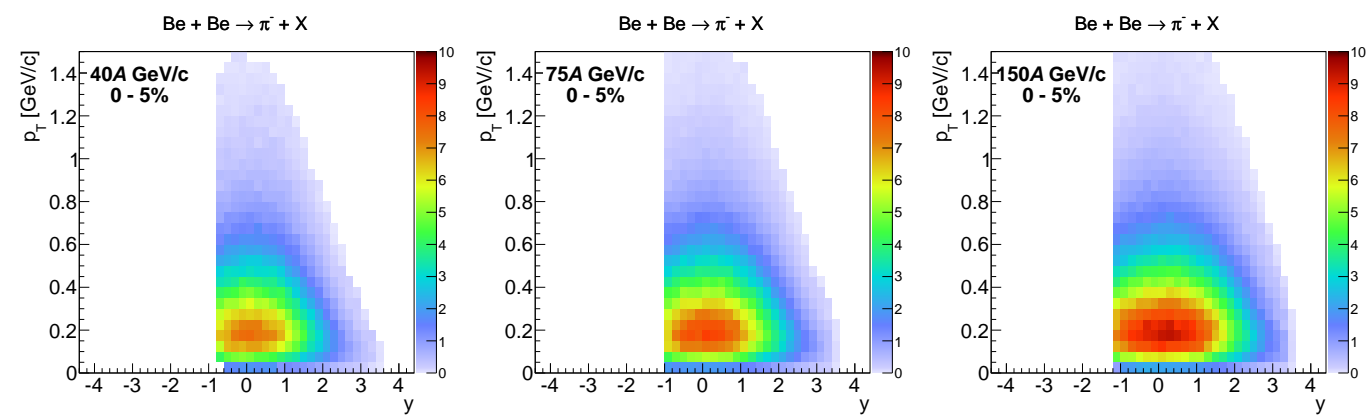

Figure 5: Double differential spectra of negatively charged pions in rapidity and transverse momentum for $\mathrm{Be}+\mathrm{Be}$ interactions at $40 \mathrm{~A}, 75 \mathrm{~A}$ and $150 \mathrm{~A} \mathrm{GeV} / \mathrm{c}$, for the first centrality class $(0-5 \%)$.

\subsection{Rapidity distributions}

The rapidity spectra in ${ }^{7} \mathrm{Be}+{ }^{9} \mathrm{Be}$ collisions at the three beam momenta and four centrality classes together with data for inelastic $\mathrm{p}+\mathrm{p}$ interactions are presented in Figure 7. One can observe a small asymmetry in the rapidity distribution for ${ }^{7} \mathrm{Be}+{ }^{9} \mathrm{Be}$ collisions around mid-rapidity. This asymmetry may result from two effects:

- the asymmetry between projectile and target nuclei $\left({ }^{7} \mathrm{Be}\right.$ projectile on ${ }^{9} \mathrm{Be}$ target) which is expected to enhance particle production in the backward (target) hemisphere,

- the selection of central collisions which requires a small number of projectile spectators without any explicit requirement imposed on the number of target spectators; this selec- 

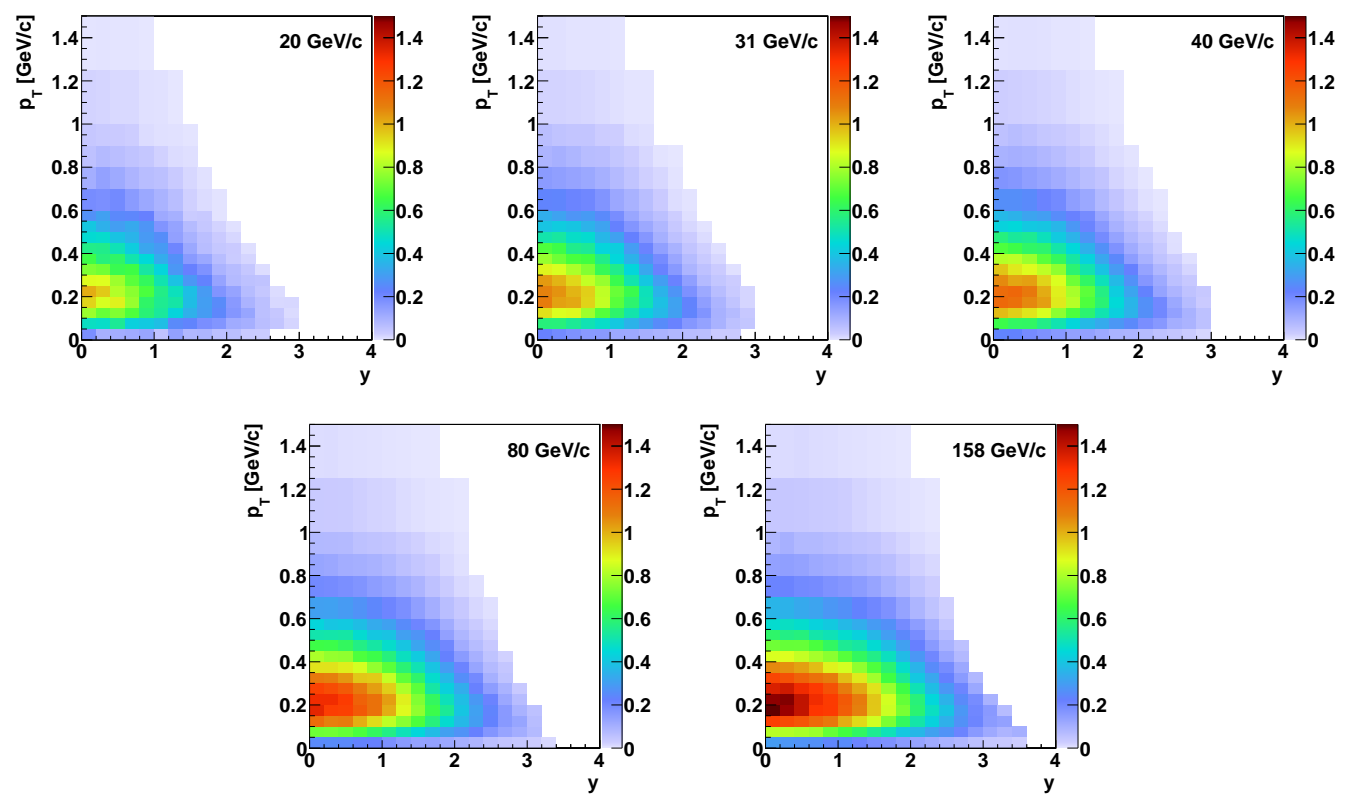

Figure 6: Double differential spectra of $\pi^{-}$mesons produced in inelastic $p+p$ interactions at 20, 31, 40, 80 and $158 \mathrm{GeV} / \mathrm{c}$.

tions used for collisions of identical nuclei would enhance particle production in the forward hemisphere.

Note that the two effects tend to compensate each other leading to a relatively small asymmetry of the measured spectra. Figure 7 shows that the second effect wins.
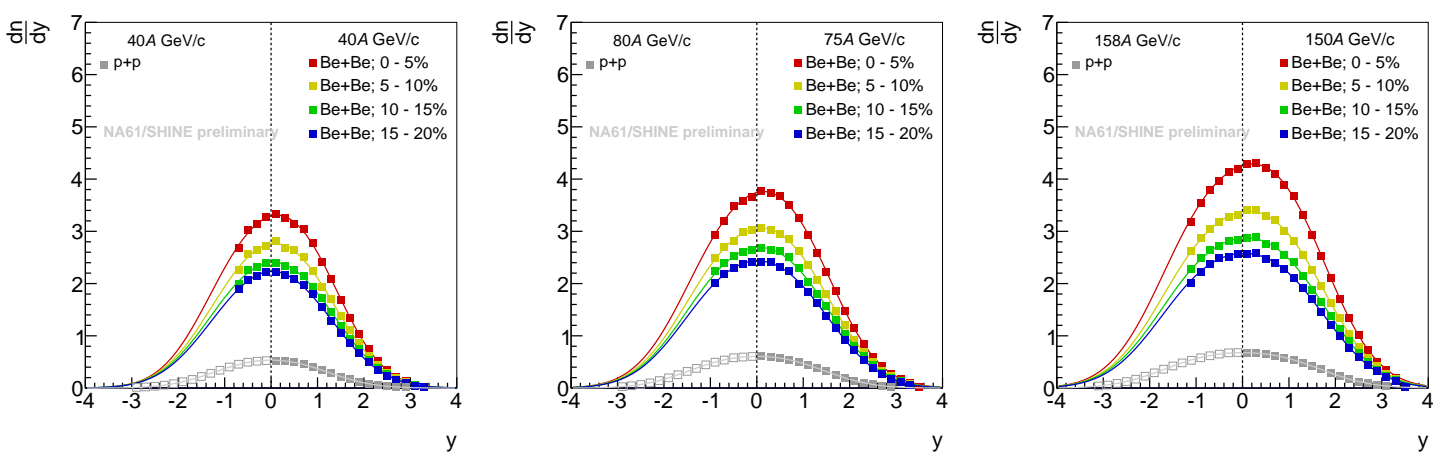

Figure 7: Rapidity density spectra of negatively charged pions in Be+Be collisions at $40 \mathrm{~A}, 75 \mathrm{~A}$ and $150 \mathrm{~A} \mathrm{GeVc}$ for four centrality classes. Spectra for inelastic $\mathrm{p}+\mathrm{p}$ interactions are shown for comparison. Note the asymmetric shape of the most central Be+Be spectra.

\subsection{Particle ratios in inelastic $p+p$ collisions}

The excellent particle identification based on the tof $-d E / d x$ method allow us to calculate $K^{+} / \pi^{+}$and $K^{-} / \pi^{-}$ratios. The energy dependence of the $K^{+} / \pi^{+}$and $K^{-} / \pi^{-}$ratios at midrapidity for inelastic $\mathrm{p}+\mathrm{p}$ interactions and central $\mathrm{Pb}+\mathrm{Pb} / \mathrm{Au}+\mathrm{Au}$ collisions is presented in Figure 8 . The NA61/SHINE data suggest that even in inelastic $p+p$ interactions the energy dependence of the 
$K^{+} / \pi^{+}$ratio exhibits rapid changes in the SPS energy range. However, the horn structure [6] is significantly reduced/modified compared to that observed in central $\mathrm{Pb}+\mathrm{Pb}$ collisions. Additionally world data $[7,8,9,10,11]$ is plotted to establish the trend beyond the SPS energy range.
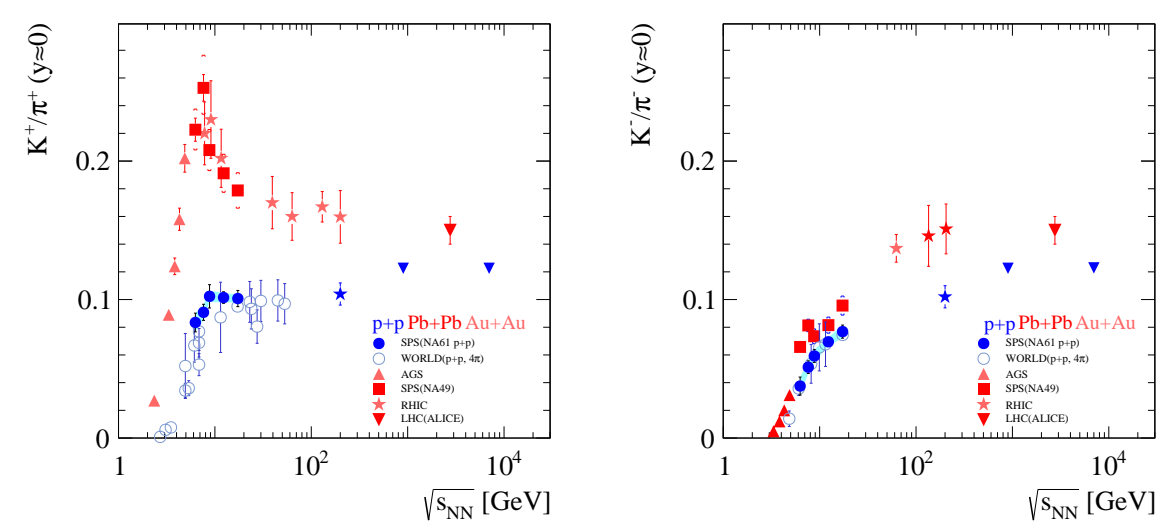

Figure 8: The $K^{+} / \pi^{+}$and $K^{-} / \pi^{-}$ratios in inelastic $\mathrm{p}+\mathrm{p}$ and central $\mathrm{Pb}+\mathrm{Pb} / \mathrm{Au}+\mathrm{Au}$ reactions plotted versus collision energy.

\subsection{Inverse slope parameter $T$}

The Inverse slope parameter $\mathrm{T}$ was fitted to the transverse mass spectra in $\mathrm{p}+\mathrm{p}$ and ${ }^{7} \mathrm{Be}+{ }^{9} \mathrm{Be}$ collisions. Figure 9 presents the energy dependence of $\mathrm{T}$ for kaons in $\mathrm{p}+\mathrm{p}$ collision. The NA61/SHINE results from inelastic $\mathrm{p}+\mathrm{p}$ collisions exhibit rapid changes like observed in central $\mathrm{Pb}+\mathrm{Pb}$ interactions. World data for $\mathrm{p}+\mathrm{p}$ and $\mathrm{Pb}+\mathrm{Pb} / \mathrm{Au}+\mathrm{Au}$ reactions are plotted for comparison and were taken from Refs. [10, 12, 13, 14].
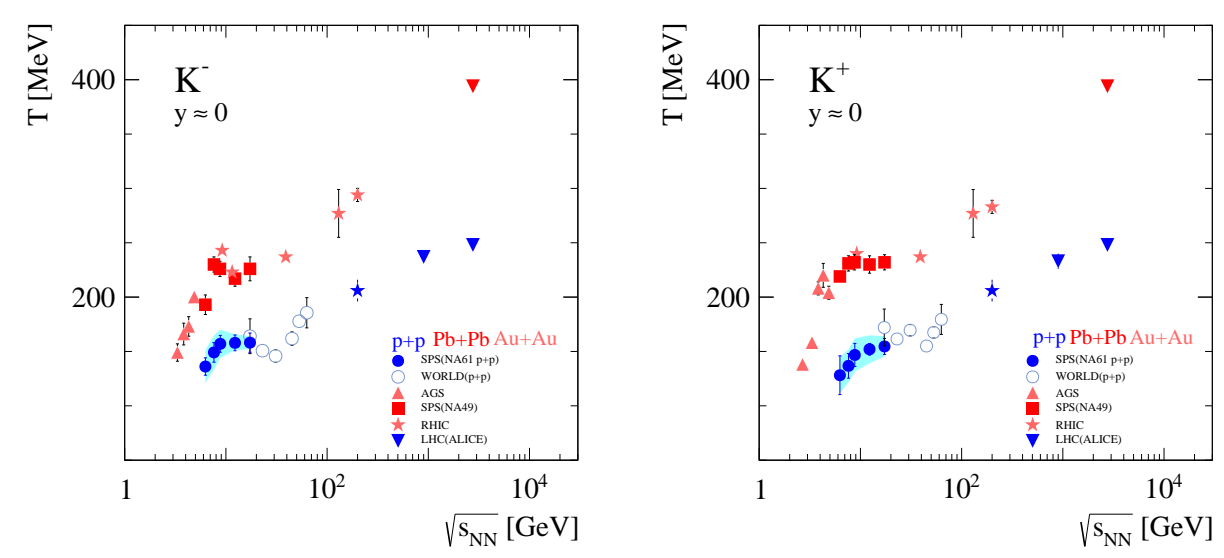

Figure 9: Energy dependence of the inverse slope parameter $\mathrm{T}$ of transverse mass spectra of kaons.

For ${ }^{7} \mathrm{Be}+{ }^{9} \mathrm{Be}$ collisions the collision energy and system size dependence of the inverse slope parameter $\mathrm{T}$ is presented in Figure 10. Points for central $\mathrm{Pb}+\mathrm{Pb}$ collisions are significantly above the results from inelastic $\mathrm{p}+\mathrm{p}$ interactions, suggesting that collective flow in $\mathrm{Pb}+\mathrm{Pb}$ collisions leads to an increase of the $\mathrm{T}$ parameter. The Be data points are between those from $\mathrm{p}+\mathrm{p}$ and central $\mathrm{Pb}+\mathrm{Pb}$ reactions. 


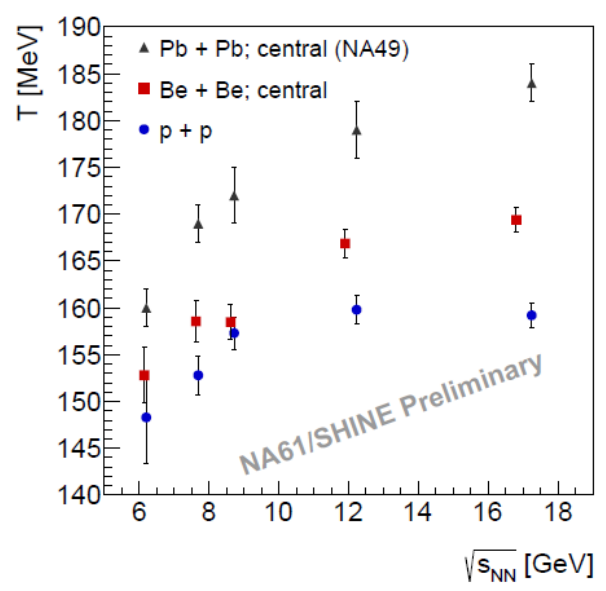

Figure 10: System size and energy dependence of the inverse slope parameter of transverse mass spectra.

\section{6 $\Lambda$ hyperon production in inelastic $p+p$ interactions at $158 \mathrm{GeV} / \mathrm{c}$}

The $\Lambda$ rapidity distribution measured by NA61/SHINE is compared to previous measurements in Figure 11. In order to partly compensate for differences resulting from different collision energies the comparison is done in terms of the scaled rapidity $z=y / y_{\text {beam }}$ and the spectra are normalized to unity. The mean $\Lambda$ multiplicity is calculated by summing up the measured rapidity spectrum and extrapolating it to lower and higher rapidities using the parametrization of the world data from Figure 11. The collision energy dependence of the mean $\Lambda$ multiplicity including the new NA61/SHINE result is plotted in Figure 12.

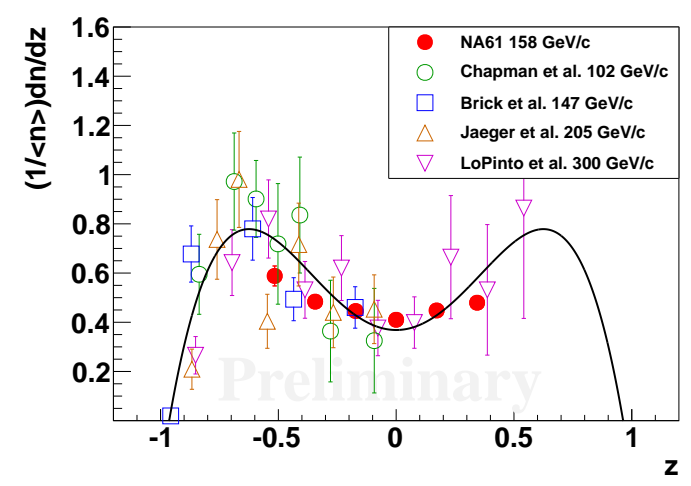

Figure 11: Scaled rapidity distribution $d n / d z\left(z=y / y_{\text {beam }}\right)$ of $\Lambda$ hyperons produced in inelastic $\mathrm{p}+\mathrm{p}$ interactions at SPS energies. The NA61/SHINE results are compared with the world data $[16,15,17,18]$. The four datasets are fitted with $\frac{1}{<n>} \frac{d n}{d z}=0.369+2.10 z^{2}-2.69 z^{4}[19]$.

\subsection{Transverse momentum fluctuations in ${ }^{7} \mathrm{Be}+{ }^{9} \mathrm{Be}$ collisions}

As an example of the NA61/SHINE results for transverse momentum fluctuations the strongly intensive measure $\Sigma\left[P_{T}, N\right]$ for ${ }^{7} \mathrm{Be}+{ }^{9} \mathrm{Be}$ interactions of several collision centralities and energies is presented in Figure 13. The results are compared with measurements of NA61/SHINE from the energy scan of inelastic $\mathrm{p}+\mathrm{p}$ interactions. 


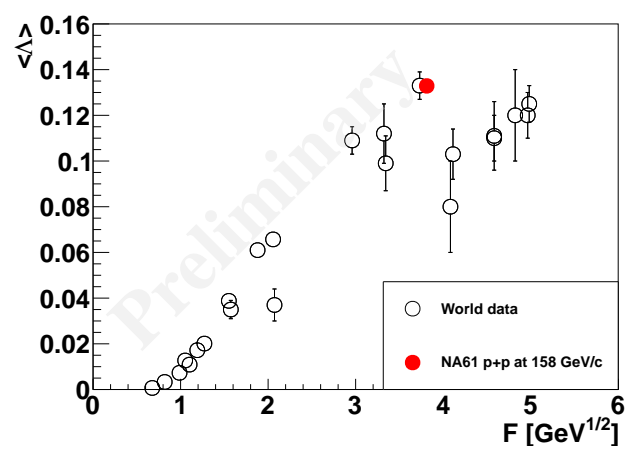

Figure 12: Mean multiplicity of $\Lambda$ hyperons produced in inelastic $p+p$ interactions as a function of the Fermi collision energy. The NA61/SHINE result is indicated by the closed point. World data are taken from compilation Ref. [8].
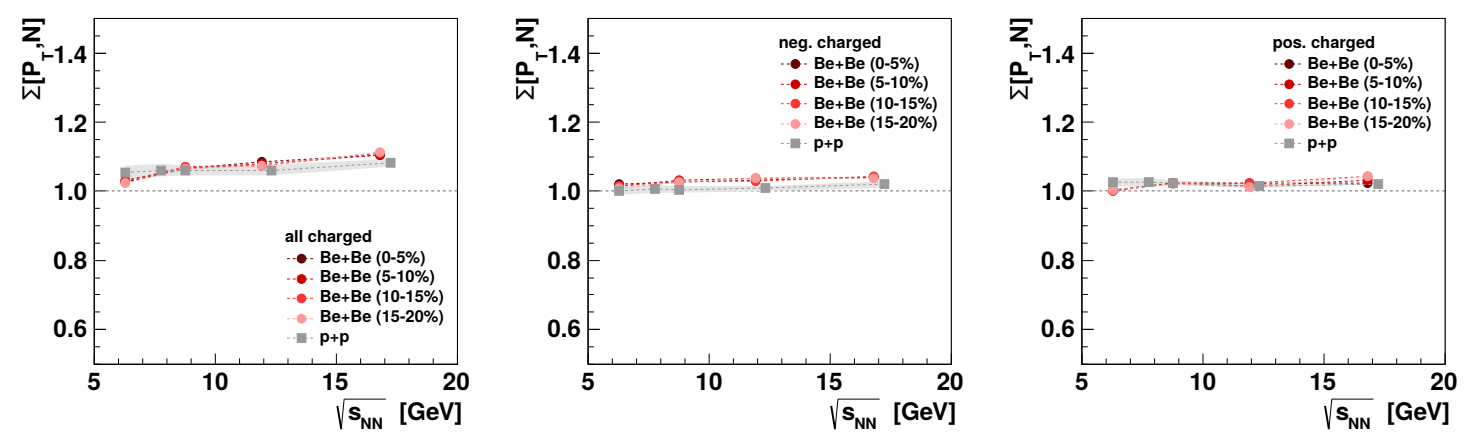

Figure 13: $\Sigma\left[P_{T}, N\right]$ for Be+Be collisions of several centralities and energies compared with measurements of NA61/SHINE from the energy scan of inelastic $p+p$ interactions.

Transverse momentum fluctuations in ${ }^{7} \mathrm{Be}+{ }^{9} \mathrm{Be}$ and $\mathrm{p}+\mathrm{p}$ interactions show no structures which could be related to the Critical Point. ${ }^{7} \mathrm{Be}+{ }^{9} \mathrm{Be}$ results are close to those from inelastic $\mathrm{p}+\mathrm{p}$ reactions.

\section{Summary}

NA61/SHINE experiment at the CERN SPS is a unique facility which operates with various primary and secondary beams (hadrons, ions) interacting in fixed targets.

NA61/SHINE pursues different programs: particle production measurements for neutrino and cosmic-ray experiments, studies of ion collisions and investigations of nuclear effects in low and high transverse momentum particle production.

High precision double-differential pion spectra were measured in inelastic $\mathrm{p}+\mathrm{p}$ reactions and in ${ }^{7} \mathrm{Be}+{ }^{9} \mathrm{Be}$ collisions at five different energies. The NA61/SHINE data suggest that even in inelastic $\mathrm{p}+\mathrm{p}$ interactions the energy dependence of the $\mathrm{K}^{+} / \pi^{+}$ratio and the energy dependence of the inverse slope parameter $\mathrm{T}$ of kaons exhibit rapid changes similar to those observed in central $\mathrm{Pb}+\mathrm{Pb}$ interactions in the SPS energy range. Moreover collective effects are observed in ${ }^{7} \mathrm{Be}+{ }^{9} \mathrm{Be}$ reactions. 
No sign of any anomaly is found in $\mathrm{p}+\mathrm{p}$ and ${ }^{7} \mathrm{Be}+{ }^{9} \mathrm{Be}$ interactions that can be attributed to the critical point.

\section{Acknowledgements}

This work was supported by the polish National Science Centre (grant 2012/04/M/ST2/00816), the Hungarian Scientific Research Fund (grants OTKA 68506 and 71989), the Polish Ministry of Science and Higher Education (grants 667/N-CERN/2010/0, NN 202484339 and NN 20223 1837), the Federal Agency of Education of the Ministry of Education and Science of the Russian Federation (grant RNP 2.2.2.2.1547), the Russian Academy of Science and the Russian Foundation for Basic Research (grants 08-02-00018,09-02-00664 and 12-02-91503-CERN), the Ministry of Education, Culture, Sports, Science and Technology, Japan, Grant-in-Aid for Scientific Research (grants 18071005, 19034011, 19740162, 20740160 and 20039012), the German Research Foundation (grant GA 1480/2-1), Bulgarian National Scientific Fondation (grant DDVU 02/19/2010), Ministry of Education and Science of the Republic of Serbia (grant OI171002), Swiss Nationalfonds Foundation (grant 200020-117913/1) and ETH Research Grant TH-01 07-3.

\section{References}

[1] N. Abgrall et al., JINST 9, P06005 (2014)

[2] N. Abgrall et al., Eur.Phys.J. C 74, 2794 (2014)

[3] I. Weimer, https://edms.cern.ch/file/1308546/1

[4] I. Tanihata et al., Phys. Rev. Lett. 55, 2676 (1985)

[5] W. Broniowski, M. Rybczynski and P. Bozek, Comput. Phys. Commun. 180, 69 (2009)

[6] C. Alt et al., Phys. Rev. C 77, 024903 (2008)

[7] M. Gazdzicki and D. Roehrich, Z. Phys. C 65, 215-223 (1995)

[8] M. Gazdzicki and D. Rohrich, Z. Phys. C 71, 55-64 (1996)

[9] I. Arsene et al., Phys. Rev. C 72, 014908 (2005)

[10] K. Aamodt et al., Eur. Phys. J. C 71, 1655 (2011)

[11] B. Abelev et al., Phys. Rev. Lett. 109, 252301 (2012)

[12] M. Kliemant, B. Lungwitz and M. Gazdzicki, Phys. Rev. C 69, 044903 (2004)

[13] B.I. Abelev et al., Phys. Rev. C 79, 034909 (2009)

[14] B.B. Abelev et al., Phys. Lett. B 736, 196-207 (2014)

[15] D. Brick et al., Nucl. Phys. B 164, 1 (1980)

[16] J.W. Chapmanet al., Phys. Lett. B 47, 465-468 (1973)

[17] K. Jaeger et al., Phys. Rev. D 11, 2405 (1975)

[18] F. LoPinto et al., Phys. Rev. D 22, 573-581 (1980)

[19] M. Gazdzicki and O. Hansen, Nucl. Phys. A 528, $754-770$ (1991) 